\title{
EXPERIMENTAL INVESTIGATIONS OF MICROWAVE EFFECTS ON ROCK BREACKAGE USING SEM ANALYSIS
}

\author{
K. Teimoori ${ }^{1}$, F. Hassani ${ }^{1}$, A.P. Sasmito ${ }^{1}$, and S.A. Ghoreishi-Madiseh ${ }^{2}$, \\ ${ }^{I}$ Department of Mining and Materials Engineering, McGill University, 3450 University, \\ Frank Dawson Adams Bldg., Montreal, QC H3A OE8, Canada \\ ${ }^{2}$ Norman B Keevil Institute of Mining Engineering, University of British Colombia, \\ Vancouver, BC, V6T 1Z4, Canada \\ khashayar.teimoori@mail.mcgill.ca \\ ferri.hassani@mcgill.ca \\ agus.sasmito@mcgill.ca \\ ali.madiseh@ubc.ca
}

\begin{abstract}
Preconditioning of hard rocks by microwave energy has recently been considered a potentially effective technology in mechanical rock breakage for civil and mining engineering. To obtain the amount of mechanical damage that a single-mode microwave treatment produces in rocks, it is necessary to analyze and evaluate the thermal cracking process by microwave heating at different power levels, exposure times, and distances from the antenna. The current study employs the scanning electron microscopy imaging technique to capture images from surfaces of irradiated rock specimens and to compare them with a nontreated specimen. To evaluate and quantify the amount of cracking (i.e. crack density, crack size, etc.) in a rock specimen after microwave irradiation with different microwave input operating parameters, the following steps were evaluated. First, several experiments of single-mode microwave treatments with different operating parameters were performed on rectangular specimens of basalt. Then, cylindrical core samples with a dimension of $\mathrm{r}=0.5 \mathrm{~cm}, \mathrm{~h}=2 \mathrm{~cm}$, were drilled from the center of the irradiated specimens and prepared for image processing. ${ }^{1}$ The results of the present study show that there are significant differences between the number of microcracks present in samples irradiated at different power levels and distances from the antenna. Also, longer exposure times result in more severe cracks.
\end{abstract}

Keywords: microwave-assisted rock breakage, rock preconditioning, scanning electron microscopy (SEM), image analysis, single-mode microwave, basalt

\section{Introduction}

Microwave applications have primarily been investigated for mineral processing to potentially reduce the required energy consumption during comminution and to increase mechanical stresses through the particles of rocks for easier separation of rock pieces [1]. The technology of applying microwave irradiation on hard rocks has recently been assessed as a potential approach for rock fracturing prior to breakage by mechanical means such as the Tunnel Boring Machine (TBM) [2]. The purpose of microwave treatment of hard rocks is to reduce their mechanical strength by weakening key mechanical and physical properties. This process is referred to as "rock preconditioning". A conceptual illustration of microwave-assisted rock breakage as an alternative to conventional methods is shown in Fig. 1.

\footnotetext{
${ }^{1}$ In this study, the term 'specimen' refers to rectangular basalt extractions of size $40 \mathrm{~cm} \times 40 \mathrm{~cm}$, while the term 'sample' refers to cylindrical basalt extractions of size $\mathrm{r}=0.5 \mathrm{~cm}$ from the center of the irradiated specimens.
} 
Understanding the complex process of rock breakage by microwave energy requires a careful consideration of various aspects. To identify the effects of applying microwave treatments at different power levels, exposure times, and distances from the antenna, a powerful microanalysis technique should be employed. Among different available methods for analyzing the number of microcracks produced in a rock specimen by microwave treatment, the scanning electron microscopy (SEM) technique is comparatively more useful for analyzing microstructural characteristics because the SEM instrument has a greater depth of field and high spatial resolution [3].

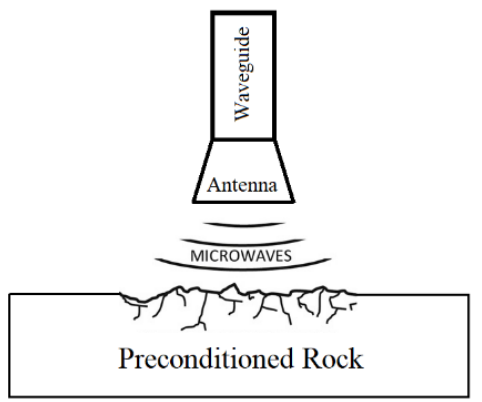

Fig. 1. The concept of microwave-assisted rock breakage

Many studies on the mechanical behavior of rocks verify that the effect of microwave treatment on hard rocks is significant [4,5]. For example, since 2005, several studies on microwave-assisted rock breakage have been conducted by the McGill microwave research team. They introduced the application of microwave energy which is considered a novel technique for rock breakage, in rock drilling, cutting, and breaking. It was shown that high-power microwave treatment resulted in an increased temperature and, therefore, the formation of mechanical damages to the rock surface, better facilitation of breakage operations for the mechanical disc cutter of an excavation machine, and reduction of disc cutter wear [4-7]. These results opened a new horizon for future breakage operations by applying microwave energy on one of the available rock excavation machines, i.e. full-face TBM. In another recent study, the authors of this paper investigated the influences of different microwave operating parameters, such as power level, exposure time, and the distance from the antenna (for single-mode microwave), on the surface temperatures of hard rocks by comparing results obtained through experiments and numerical simulations. Their results demonstrated that microwave energy has the potential to damage hard rocks by inducing multiple microcracks [6].

Although thermal stress and thermal cracking mechanisms by microwaves have been studied for many years, microstructural analysis of the processes of crack initiation, intensity, and their relevance to the applied microwave operating parameters has not been extensively carried out. Therefore, this challenge needs to be addressed through a fundamental understanding of how microwaves interact with hard rocks. It is essential to explore comprehensively the microstructural characteristics of rocks before and after microwave treatments using a more accurate analysis. The current study investigates and analyzes the amount of damage produced by microwave treatments on basalt specimens with different power levels, distances from the antenna, and exposures. By taking account of the features of the scanning electron microscopy imaging technique, the present study investigates the microstructural behavior of basaltic rock specimens after microwave treatments by using the captured images from the SEM device. 


\section{Methodology}

The method utilized in this study involves the following four steps:

1- Microwave irradiation experiments with different operating parameters on rectangular specimens of basalt,

2- Preparing cylindrical core samples from the center of the irradiated basalt specimens using a drill bit with an inner radius of $0.5 \mathrm{~cm}$,

3- Image processing on the cylindrical cored samples using an SEM instrument,

4- Crack quantification analysis on the SE images using ImageJ software.

The experimental tests were performed in the following steps. First, multiple rectangular basalt specimens were placed together in a series on an insulator slab inside a single-mode microwave cavity to arrive at the desired distance from the antenna (see Fig. 2b). Then, microwave irradiation was operated at different experimental conditions for a total of twelve case studies. The input operating parameters of the microwaves for the experiments are given in Table 1. For better evaluation of the impact of the different microwave operating parameters on microcracking behavior, case no. 1 was designated a nontreated basalt sample.

\begin{tabular}{cccc}
\hline Case no. & Power $(\mathrm{KW})$ & Distance from the antenna $(\mathrm{cm})$ & Exposure time $(\mathrm{s})$ \\
\hline 1 & nontreated & nontreated & nontreated \\
2 & 3 & 5 & 60 \\
3 & 3 & 15 & 60 \\
4 & 3 & 20 & 60 \\
5 & 3 & 25 & 60 \\
6 & 9 & 15 & 60 \\
7 & 9 & 20 & 60 \\
8 & 9 & 25 & 60 \\
9 & 15 & 20 & 60 \\
10 & 15 & 25 & 60 \\
11 & 3 & 5 & 120 \\
12 & 9 & 15 & 120 \\
\hline
\end{tabular}

Table 1. Input operating parameters of microwave for the experiments

By referring to a recent paper by the authors [6], the current study by performing crack quantification analysis provides a valuable way to understand the changes in the microstructure of the basalt rock specimen after microwave irradiation with different operating parameters. When specimens of basalt were subjected to different microwave treatments, it was expected that several microcracks would be induced because of the increased thermal expansion inside the irradiated specimens. However, the intensity of microcracks was highly dependent on the input parameters of the applied microwaves. The results from crack quantification analysis on irradiated cylindrical cored samples were compared with the nontreated sample.

\subsection{Microwave treatment experiments}

Microwaves are designed in two different types as follows: single-mode and multimode. Single-mode microwaves consist of a metallic enclosure and a waveguide horn. These metallic parts were designed so that the electromagnetic field polarization from a microwave signal would undergo several reflections. The superposition of the propagated waves in different directions gives rise to a standing wave pattern [8]. This pattern of waves is very well defined inside the cavity's space. Consequently, the precise knowledge of different 
configurations of the electromagnetic waves enables the rock specimen to be placed in the position at which maximum heating rate and subsequent breakage can be achieved. With that information in mind, a single-mode microwave cavity was chosen for the experiments of the present study. An illustration of this single-mode cavity system is given in Fig. 2.

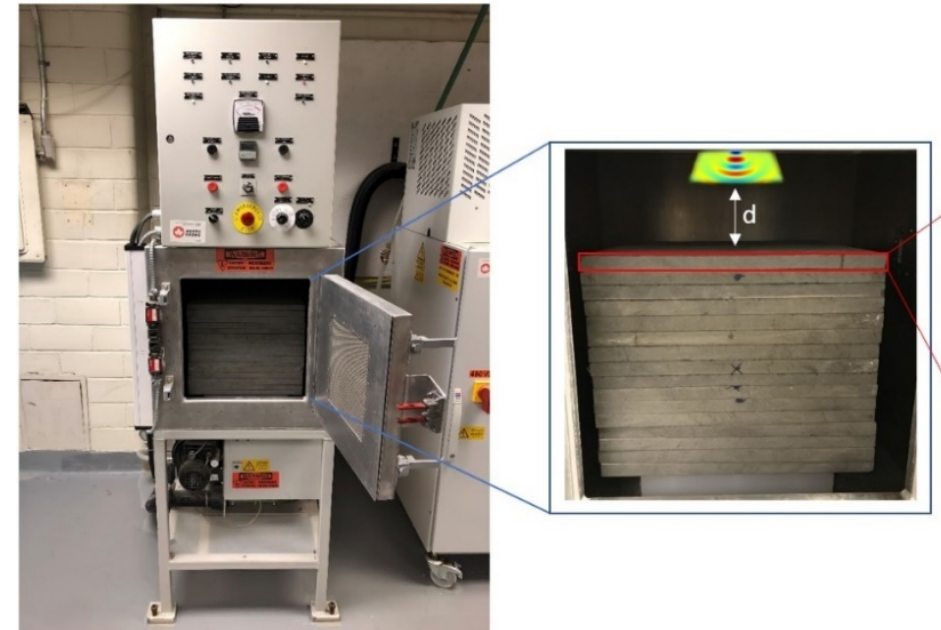

(a) (b)

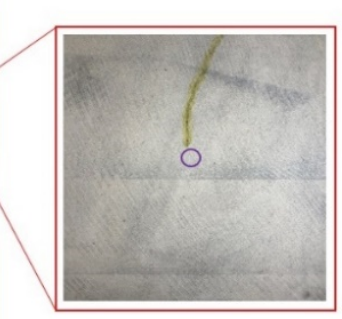

(c)

Fig. 2. (a) Single-mode microwave cavity system, (b) Microwave equipment set-up with the rock specimens placed inside the microwave cavity and below the horn; the distance from the waveguide horn (antenna), $\mathrm{d}(\mathrm{cm})$, is shown by a white arrow, and (c) the first irradiated specimen from top after the experiment. Note here that the first specimen from the top is used for drilling cored samples from its center (shown by a purple circle).

The blocks of basalt were cut at the mine into rectangular specimens with dimensions of $40 \mathrm{~cm} \times 40 \mathrm{~cm}$. The specimens were intact and without visible cracks. They were used to conduct microwave tests according to the input conditions of Table 1. After the experiments, the first specimen from the top was used for the drilling of cylindrical core samples (see Fig. 2(c)). Subsequently, the SEM instrument was employed to capture SE images from the top center of the cored samples and for further image processing purposes. Finally, crack quantification analysis was performed on the rendered images using ImageJ software. The results were used to compare the effects of different microwave operating conditions and to analyze the microcracking behavior of basalt.

\subsection{Core samples' preparation process}

After microwave treatment tests, the irradiated specimens were drilled using a drill bit with an inner radius of $r=0.5 \mathrm{~cm}$; cylindrical core samples, with a dimension of $r=0.5 \mathrm{~cm}$ and $h=2 \mathrm{~cm}$, were drilled into the surface of the irradiated specimens for SEM image analysis. The drill bit and cored samples' size are shown in Fig. 3(a) and 3(b), respectively. For a better visual understanding of the SEM imaging process, the SEM device (type SU3500) is illustrated in Fig. 3(c). 


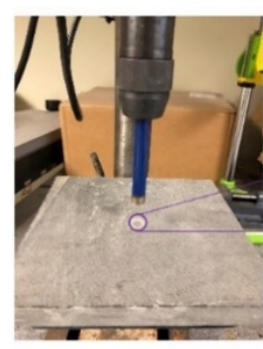

(a)

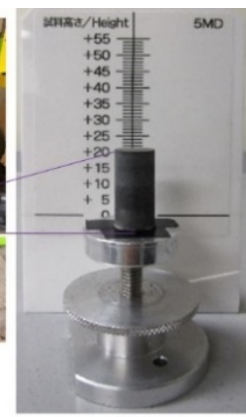

(b)

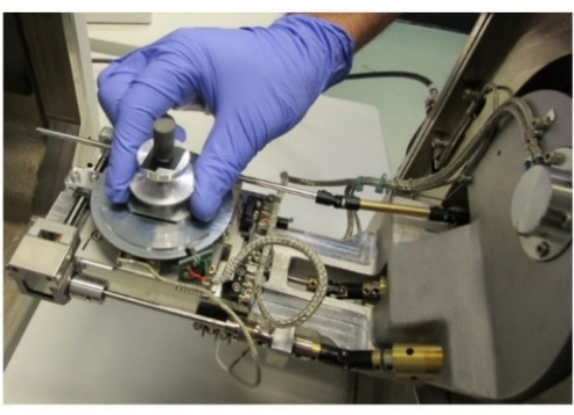

(c)

Fig. 3. (a) The drill bit for making core samples, (b) cylindrical cored samples with a dimension of $\mathrm{r}=0.5 \mathrm{~cm}$ and $\mathrm{h}=2 \mathrm{~cm}$, and (c) installation process of the core sample in SEM-SU3500 instrument for image processing

\subsection{SEM analysis}

Scanning electron microscopy (SEM) is one of the most advanced technologies for the quantification and analysis of microstructural responses of solid materials. SEM analysis is comparatively more useful in studies that investigate quantification of microstructural properties such as microcracks and voids. Fig. 4 shows the Hitachi SEM SU3500 instrument used for SEM imaging.

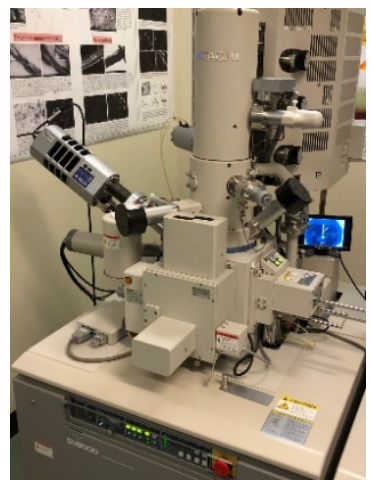

(a)

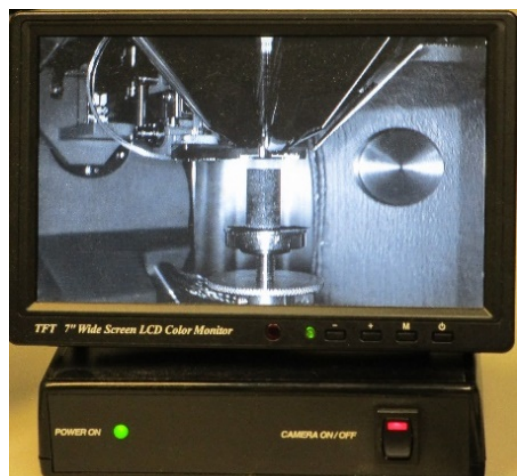

(b)

Fig. 4. (a) The Hitachi SEM-SU3500 Variable Pressure Scanning Electron Microscope (VP-SEM) instrument located at McGill University, Department of Mining and Materials Engineering, (b) position monitoring of sample

The Hitachi SU3500 Variable Pressure Scanning Electron Microscope (VP-SEM) used in the present study can provide up to $7 \mathrm{~nm}$ SE images with a resolution at $3 \mathrm{kV}, 10 \mathrm{~nm}$, and backscattered electrons (BSE) image resolution at $5 \mathrm{kV}$. It is equipped with an $80 \mathrm{~mm}$ 2X-Max SDD EDS detector with the EBSD Aztec HKL Advanced system. The high probe of this microscope with $1 \mu \mathrm{A}$ current allows the automatic EDS-EBSD mapping to create a montage of all the regions of a given specimen automatically at very high speed [9]. The BSE detector can produce quantitative three-dimensional maps of the surface of a specimen to quantify complicated and rough surfaces. The low vacuum mode characterizes nonconductive materials without coatings.

In this study, the SE images were captured with a resolution at $2 \mathrm{kV}$ and $200 \mu \mathrm{m}$. After acquiring the images from the irradiated specimens with the conditions given in Table 1, image processing was performed to view microcracks in basalt. 
ImageJ software developed by the National Institute of Health (NIH) was used to conduct image processing [10]. This software has the capability to perform various operations such as binary thresholding and area measurement based on Java language.

\section{Results and discussion}

The results from the SE images are shown in Fig. 5. The plots follow the case studies listed in Table 1. The specified case numbers are shown under each plot. It can be seen that the intensity of microcracks reduces when the power level decreases from $15 \mathrm{KW}$ to $3 \mathrm{KW}$, the exposure time decreases from 120 -second to 60 -second, and the distance from the antenna increases from $5 \mathrm{~cm}$ to $25 \mathrm{~cm}$.

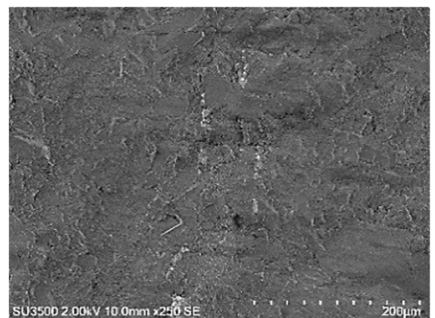

Case no.1

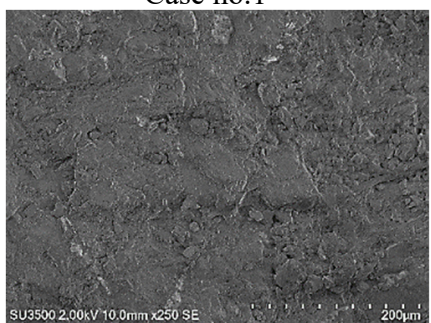

Case no. 4

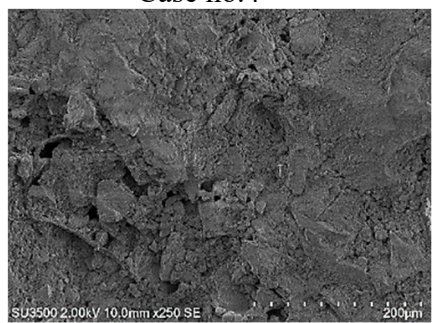

Case no.7

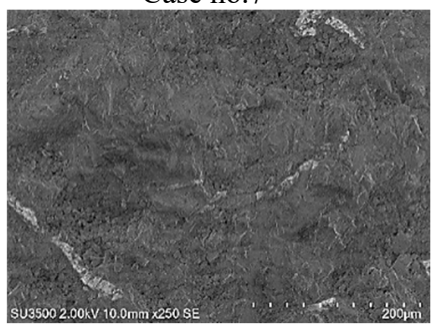

Case no.10

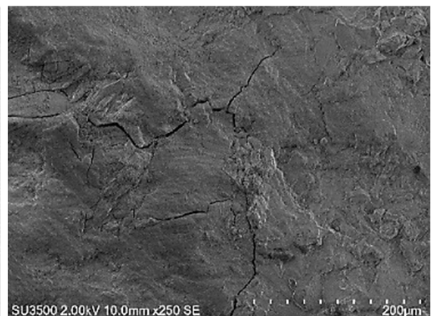

Case no. 2

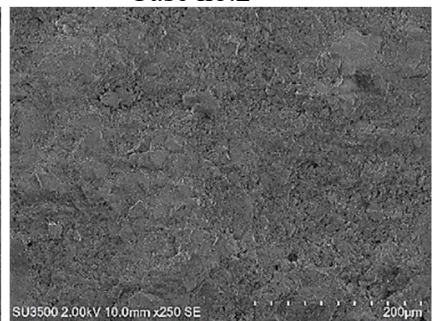

Case no.5

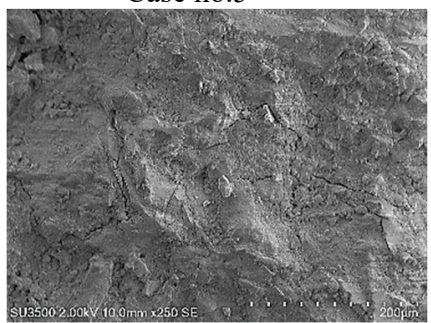

Case no. 8

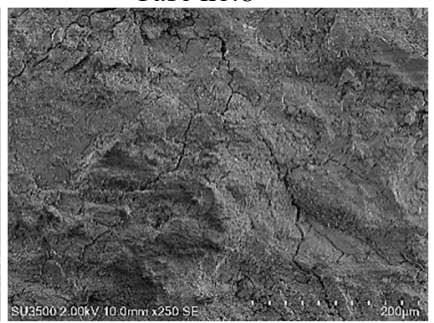

Case no.11

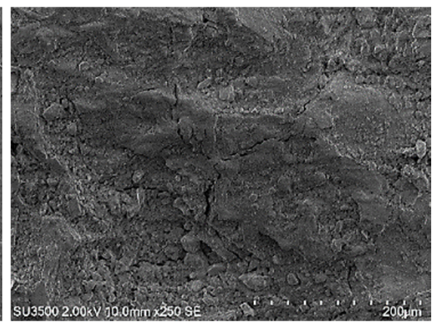

Case no. 3

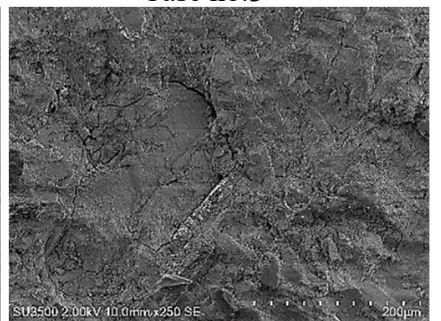

Case no.6

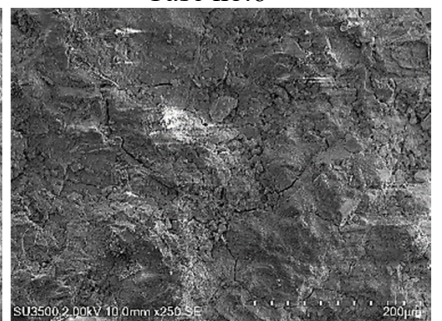

Case no. 9

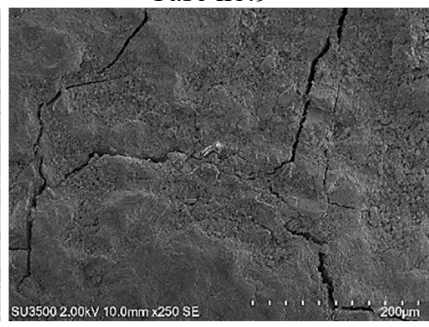

Case no. 12

Fig. 5. The SEM images of cylindrical cored samples after microwave treatments with different operating parameters 
The first step of image processing was to conduct segmentation of the images into binary images through the brightness thresholding approach. This makes the SE images black and white. By this process, ImageJ changes the intensities of image pixels that will highlight the regions of interests - in this study, are microcracks. For each plot of Fig. 5, the low threshold level was set at zero and the best threshold level was determined by comparing the original gray image with the image after application of manual thresholding on the binary image. After being converted to binary mode, all images were manually mapped, and the total number of crack lengths and crack density for all the case studies was obtained. However, it is worth mentioning here that microcracks are very elongated structures; therefore, length to width (referred to as shape factor) analysis is applied for different geometries. This means that the structures with a shape factor of 2.7 or greater were considered microcracks. In this case, the remaining structures should be assumed to be voids. The corresponding data obtained for the length and density of cracks for the different case numbers after image processing on the SE images are summarized in Table 2.

\begin{tabular}{cccc}
\hline Case no. & Cracks length $(\mu \mathrm{m})$ & Image field & Crack density $\left(\mu \mathrm{m} / \mu \mathrm{m}^{2}\right)$ \\
\hline 1 & 432.15 & $183 \mu \mathrm{m} \times 250 \mu \mathrm{m}$ & 0.009445 \\
2 & 1310.64 & $183 \mu \mathrm{m} \times 250 \mu \mathrm{m}$ & 0.028647 \\
3 & 1182.07 & $183 \mu \mathrm{m} \times 250 \mu \mathrm{m}$ & 0.025837 \\
4 & 943.74 & $183 \mu \mathrm{m} \times 250 \mu \mathrm{m}$ & 0.020628 \\
5 & 780.42 & $183 \mu \mathrm{m} \times 250 \mu \mathrm{m}$ & 0.017058 \\
6 & 1670.72 & $183 \mu \mathrm{m} \times 250 \mu \mathrm{m}$ & 0.036518 \\
7 & 1322.91 & $183 \mu \mathrm{m} \times 250 \mu \mathrm{m}$ & 0.028916 \\
8 & 1107.085 & $183 \mu \mathrm{m} \times 250 \mu \mathrm{m}$ & 0.024198 \\
9 & 1432.11 & $183 \mu \mathrm{m} \times 250 \mu \mathrm{m}$ & 0.031302 \\
10 & 1216.32 & $183 \mu \mathrm{m} \times 250 \mu \mathrm{m}$ & 0.026586 \\
11 & 1540.02 & $183 \mu \mathrm{m} \times 250 \mu \mathrm{m}$ & 0.033661 \\
12 & 1861.81 & $183 \mu \mathrm{m} \times 250 \mu \mathrm{m}$ & 0.038909 \\
\hline
\end{tabular}

Table 2. Crack quantification analysis for basalt samples

The above results indicate the presence of microcracks in the given sample. Maximum lengths of cracks were $1670.72 \mu \mathrm{m}$ and $1861.81 \mu \mathrm{m}$, for 60 -second and 120 -second exposure times, respectively. These two values were achieved when a $3 \mathrm{KW}$ power level was applied at a $5 \mathrm{~cm}$ distance from the antenna. Therefore, it can be concluded that the effect of exposure time was significant; longer exposure times were responsible for more extensive cracks. In addition, for a better understanding of the cracking mechanism, a crack quantification analysis was done on the nontreated sample compared to the treated samples. The result of the nontreated sample is shown in case no. 1 in Table 2. When comparing results from the irradiated samples with the nontreated sample, the effect of microwave input operating parameters is more visible. For example, by comparing cases 4 and 5, in which the amount of crack length was under $1000 \mu \mathrm{m}$, the effect of the distance from the antenna can be observed. As the distance from the antenna increases, the number of microcracks declines. The results from crack quantification analysis yield a better understanding of the mechanism of crack propagation in regard to differently applied microwave operating parameters. The density of cracks at the captured area differs, depending upon different microwave input operating parameters. By comparing the data in Table 2, it can be observed that a higher level of microwave power results in greater crack lengths and densities. On closer inspection, however, at the same power level, a shorter distance from the antenna results in denser cracking. 


\section{Conclusion}

A previous study conducted by the authors [6] showed that microwave energy can thermally break hard rocks by inducing several microcracks; however, the actual mechanisms in such a complex system with different multiphysics interactions are still hard to investigate. The objective of this study was to analyze the microcracking behavior of rocks under microwave treatments with different operating parameters using the SEM technique. For a better visual understanding of the mechanism of crack development, including spallation/disintegration, the SEM images were captured from the top center of the cylindrical cored samples.

The previous study also showed that rock failure mechanism under microwave irradiation tests is closely related to the input operating parameters of the applied microwave energy. More significantly, a higher input power level of microwaves contributes to more complex physical and mechanical reactions, which include the elevation of internal pressure and decomposition of particles in the basalt specimen. The results both studies are highly consistent with each other.

\section{Acknowledgements}

Financial support for this work from the Natural Sciences and Engineering Research Council of Canada (NSERC), De Beers Group, Argex, Metso and the McGill Engineering Doctoral Award (MEDA) Award is gratefully acknowledged.

\section{References}

[1] Lovas, M., Znamenackova, I., Zubrik, M., Kovacova, M., and Dolinska, S., "The Application of Microwave Energy in Mineral Processing - a Review”, Acta Montanistica Slovaca, 2011, 16(2), pp. 137-148.

[2] Lu, G. ming, Li, Y. hui, Hassani, F., and Zhang, X., “The Influence of Microwave Irradiation on Thermal Properties of Main Rock-Forming Minerals", Appl. Therm. Eng., 2017, 112, pp. 1523-1532.

[3] Nemati, K. M., "Fracture Analysis of Concrete Using Scanning Electron Microscopy”, Scanning, 2006, 19(6), pp. 426-430.

[4] Motlagh, P. N., "Physical and Mechanical Properties of Rocks Exposed: To Microwave Irradiation: Potential Application to Tunnel Boring”, McGill Univ., 2015.

[5] Hassani, F., Nekoovaght, P. M., and Gharib, N., "The Influence of Microwave Irradiation on Rocks for Microwave-Assisted Underground Excavation", J. Rock Mech. Geotech. Eng., 2016, 8(1), pp. 1-15.

[6] Teimoori, K., Hassani, F., and Sasmito, A. P., "Multiphysics Study of Microwave Irradiation Effects on Rock Breakage System”, IJRMMS, 2019, 1(514), pp. 1-32.

[7] Lu, G., Feng, X., Li, Y., Hassani, F., and Zhang, X., "Experimental Investigation on the Effects of Microwave Treatment on Basalt Heating, Mechanical Strength and Fragmentation," Rock Mech. Rock Eng., 2019, 1(Feb), pp. 1-19.

[8] Whittles, D. ., Kingman, S. ., and Reddish, D. ., "Application of Numerical Modelling for Prediction of the Influence of Power Density on Microwave-Assisted Breakage”, Int. J. Miner. Process., 2003, 68(1-4), pp. 71-91.

[9] Brodusch, N., Demers, H., and Gauvin, R., "Imaging with a Commercial Electron Backscatter Diffraction (EBSD) Camera in a Scanning Electron Microscope: A Review”, J. Imaging, 2018, 4(7), p. 88.

[10] Abramoff, M. D., Magalhães, P. J., and Ram, S. J., “Image Processing with ImagJ”, Biophotonics Int., 2004, 11(7), pp. 36-42. 